\title{
Bamboo-infoline: A database for North Bengal Bamboo's
}

\author{
Arvind Kumar Goyal ${ }^{1}$, Sushil Kumar Middha ${ }^{2}$, Talambedu Usha ${ }^{2}$, Subhojyoti \\ Chatterjee $^{2}$, Asim Kumar Bothra ${ }^{3}$, Madhugiri Bhujangrao Nagaveni ${ }^{2}$, Arnab \\ $\operatorname{Sen}^{1} *$
}

${ }^{1}$ NBU Bioinformatics Facility, Department of Botany, University of North Bengal, Siliguri- 734013, West Bengal, India; ${ }^{2}$ Maharani Lakshmi Ammanni College For Women (MLACW), DBT-BIF centre, Banglore-560012,Karnataka, India; ${ }^{3}$ Bioinformatics Chemoinformatics laboratory, Department of Chemistry, Raiganj College, Raiganj (W.B), India. Arnab Sen - Email : senarnab_nbu@hotmail.com *Corresponding author

Received August 27, 2010; Accepted September 27, 2010; Published October 06, 2010

\begin{abstract}
:
Bamboo, the "Green Gold", included in the non-timber forest products has both ecological and economic importance. Here is an attempt to describe a database named "Bambooinfoline", which provides enumeration of the different species of bamboos found in North Bengal, with special emphasis on taxonomy, edible properties, chemical constituents, morphological features along with tissue culture specifications, which in turn benefits of scientific community.
\end{abstract}

Availability : http://www.bamboodb.ind.in/

Keywords : Bamboo, North Bengal, HTML, Tissue culture, Pubmed, NCBI, Real time, Database, MeSH, Green gold

Background :

Bamboo the "poor man's timber" is the fastest growing perennial evergreen arborescent plant belonging to the true grass family Poaceae, subfamily Bambusoideae, tribe Bambuseae [1]. There are about 1,500 species under 87 genera worldwide [2], which are unevenly distributed in various parts of the humid tropical, sub-tropical and temperate regions. Vegetative growth phase among species of bamboo ranges from 1 to 120 years [3]. India is well endowed with bamboo resources, and is the second largest in the world after China [4]. In India, bamboo grows on millions of hectares (almost 8.96 million hectares) of forest land, in homesteads and on private plantations which accounts for about fifty percent of the total land area under bamboo in Asia. There are almost 136 indigenous and exotic species, found naturally and/or under cultivation in India [5], spread across 36 genera. North Bengal has a large variety of bamboos. Its wide range of uses and versatility makes it eligible to be of multiple use alternatives to timber, food for rural poor and tribal in particular. North Bengal is endowed with some important genera of Bamboo like Bambusa, Cephalostychum, Dendrocalamus, Drepanostachyum, Gigantocloa, Himalayacalamus, Melocanna, Pleioblastus, Phyllostachys, Pseudosasa, Sasaella, Shibataea, and Yushnia which include different species, subspecies and varieties. Since bamboo is considered to be a multipurpose plant having about 1500 documented uses [6], its importance has increased many folds in the last few decades.

There is no such academic public domain available on bamboo worldwide and those available are commercial. Considering the ecological and economical significance of bamboo in North Bengal, authors are making first effort to explore this region to have an insight into the distribution and diversity of bamboo, after consulting a bamboo taxonomist. The primary objective of this database is to extensively explore the published literature on bamboo taxonomy, edible properties, morphological features, along with tissue culture specifications and chemical constituents. This database is an attempt to give compiled information on various species of bamboo's encountered in this region which in turn will help the interested researchers and bamboo lovers to have an in-depth idea about each species.
Methodology :

Construction of Bamboo-infoline: [7]

Bamboo-infoline is constructed using HTML and can be accessed at http://www.bamboodb.ind.in/. Data were collected from various literature sources such as, PubMed [8], Science Direct [9], and others. A panel of technical experts representing diverse disciplines was established to advice us throughout the preparation of this database. For searching we used the MeSH terms "Bamboo" or "chemical constitutes" or "taxonomy" combined with the botanical names of different bamboo species. As a part of this, several Indian journals were surveyed to gather information. Additional articles were obtained from supplemental searches which focused on the books and review articles. All titles, abstracts, and articles were reviewed by two reviewers, and all disagreements were resolved by consensus. Data was collected using screening forms, which was developed for this purpose. We analyzed the data regarding the general characteristics or taxonomic values from the literature and used this information to construct bamboo-infoline. Figure 1 shows diversity of bamboo worldwide and in India. The complete list of 45-50 species can be found at http://www.bamboodb.ind.in/bamboomining.html. A screenshot of the Bamboo - infoline is given in Figure 1.

\section{Description of the evidence}

More than 1000 articles were searched and considered in the preparation of this database (given in reference section of database as link to PMID). Regional studies are provided to show our collection points. Maximum annotated studies came from the different published literatures as well as from the forest dwellers. All pictures incorporated in this database are original and photographed from the forest of SUKNA, after a taxonomical identification and deserve copyright by the authors. There are several commercial databases to provide commercial value and information on bamboo.Our databases, which are more academic oriented, have higher endues and will be regularly updated. Database will have direct links with original research articles, which makes our database unique from the other databases. Inputs from the authors, while seeking their permission to use their papers to update our database, provides more information to our existing database. 


\section{Bioinformation}

\section{Volume 5}

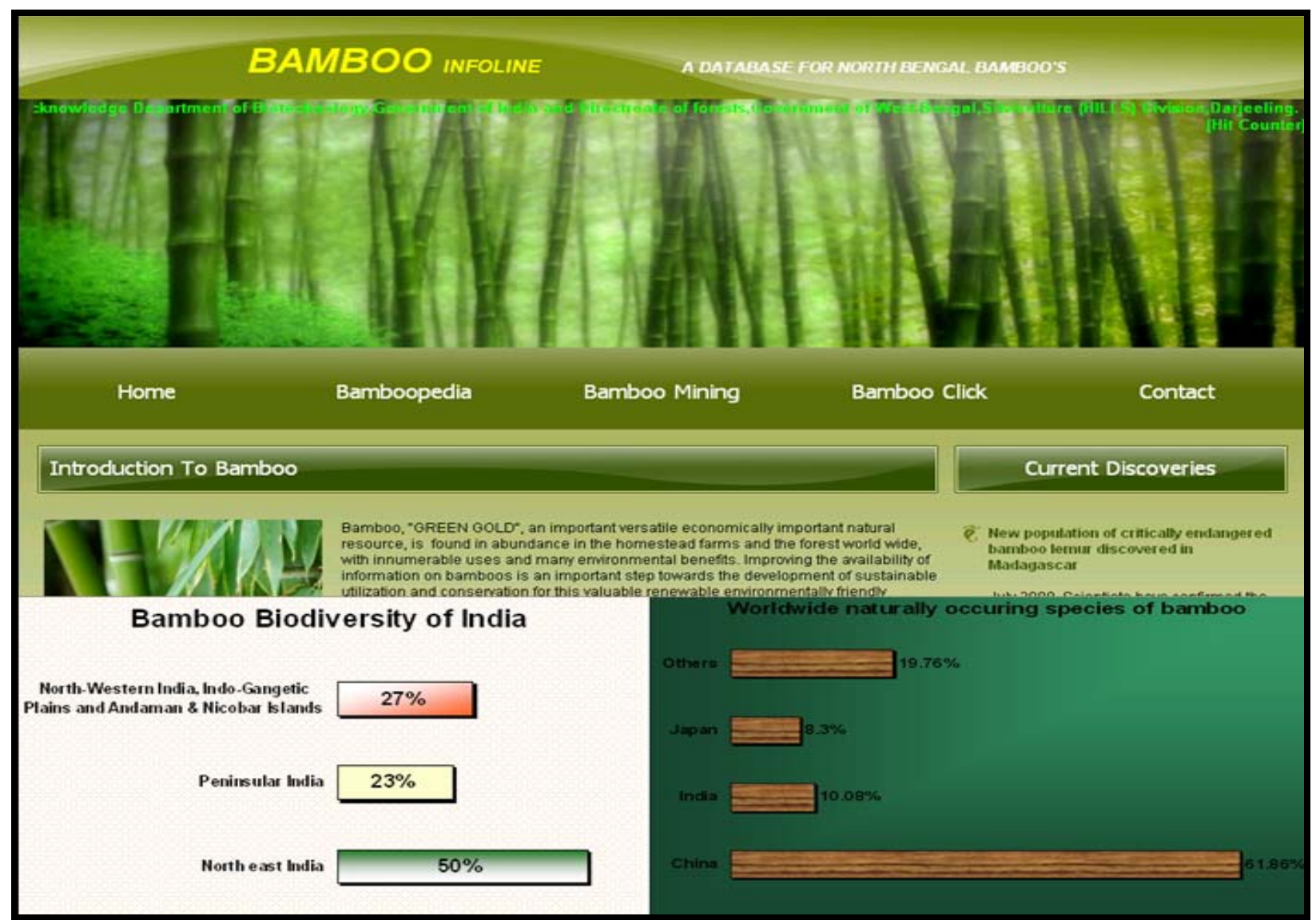

Figure 1: Snap Shot of Bamboo-infoline

\section{Utility:}

Empirical evidence of efficacy of this database for bamboo would be helpful to identify areas for future research in providing a quick review on the number of bamboo species for the benefit of scientific community and bamboo lovers.

\section{Future Development :}

Bamboo-infoline is the first approach as a free accessible academic real time database for bamboo worldwide. We are going to incorporate more scientific information regarding bamboo species of this region. Periodically continuous updates shall be released to include other bamboo species. We plan to incorporate a provision to avail the required information using keywords.

\section{Acknowledgement :}

The authors are thankful to the Department of Biotechnology, Government of India, for providing the Bioinformatics facility under BTISnet at NBU and MLACW. We are also grateful to the Mr. Ajay Kr. Dubey, DFO, and Mr. Prasanta Kr. Ghosh, Range Officer, Directorate of Forests, Government of West Bengal, Silviculture (Hills) Division, Kurseong Research Range and Dr. T.L. Shantha, Director MLACW, Bangalore for their necessary help, support and information. The authors are also obliged to the bamboo taxonomist Mr. P. P. Paudyal, for helping in identifying the species of bamboo.

\section{Conflict of interest:}

Authors don't have any conflict of interest.

\section{References :}

[1] BN Kigomo, KFRI, Eco. S., Monograph 1: 1 (1988)

[2] D Ohrnberger, Elsevier, Amsterdam. 585 p. (1999)

[3] DH Janzen, Anal Rev. Ecol.System. 7: 347 (1976)

[4] SN Rai, KVS Chauhan, Indian Forester 124 (2): 89 (1998)

[5] YML Sharma, Recent Research on Bamboos, (eds.) A.N. Rao, G. Dhanarajan and C.B. Sastry. CAF, China and IDRC, Canada, 14 (1987)

[6] JMO Scurlock Biomass and Bioenergy 19(4): 229 (2000)

[7] SK Middha et al, Bioinformation, 4 (2):78 (2009) [PMC2823386]

[8] www.ncbi.nlm.nih.gov

[9] http://www.sciencedirect.com 